\title{
APSA Contributors
}

AS OF NOVEMBER 15, 2012

This list celebrates the generous contributions of our members in giving to one or more of the following programs from 1996 through 2012: APSA awards, programs, the Congressional Fellowship Program, and the Centennial Campaign. APSA thanks these donors for ensuring that the benefits of membership and the influence of the profession will extend far into the future. APSA will update and print this list annually in the January issue of PS.

\section{CENTENNIAL CIRCLE}

$(\$ 25,000+)$

Anonymous

Walter E. Beach

Doris A. Graber

Pendleton Herring

Chun-tu Hsueh Endowment for International Scholars and Huang Hsing Foundation

Arend Lijphart

Elinor and Vincent Ostrom

Policy Studies Organization

Estate of Leo A. Shifrin

Frank J. Sorauf

University of Louisville

\section{CIRCLE}

$(\$ 15,000+)$

Richard F. Fenno, Jr.

Jo Freeman

Robert O. and Nannerl Keohane

Norman H. Nie

Jack W. Peltason

University of Iowa, Office of the Provost

Stephen L. Wasby

\section{CIRCLE}

$(\$ 10,000+)$

David S. Broder

Roger H. and Nancy Davidson

Heinz Eulau

Ruth S. Jones and Warren E. Miller

Martha Joynt Kumar

Dale Rogers Marshall

Kenneth J. Meier

Northwestern University, Office of the President
Pi Sigma Alpha

Robert D. Putnam

Ron and Rosemary Schmidt

Smith College, Government Department

Janet D. Steiger

\section{FOUNDERS CIRCLE}

$(\$ 5,000+)$

Anthony Affigne

Barbara B. Bardes

Lucius J. Barker

Robert H. Bates

Susan C. Bourque

David W. Brady

John G. Bretting

Robert Durant

Luis Ricardo Fraga

James L. Gibson

Betty Glad

John Mark Hansen

Robert J-P. Hauck

Martin O. Heisler

Jennifer L. Hochschild and C. Anthony Broh

Matthew Holden, Jr.

Robert A. Horn

Samuel P. Huntington

Gary C. Jacobson

Malcolm E. Jewell and Sarah M. Morehouse

Joyce K. Kallgren

Mary Fainsod Katzenstein and Peter J. Katzenstein

Ira Katznelson

Sydnee Guyer Lipset

Theodore J. Lowi

Valerie J. Martinez-Ebers

Paula D. McClain and Paul Jacobson
Cynthia McClintock

Ruth P. Morgan

Norman J. Ornstein

T.J. Pempel

Dianne M. Pinderhughes

Jewel L. Prestage

Lucian W. Pye

J. Austin and Nancy Ranney

Catherine E. Rudder

Eric J. Scott

J. Merrill Shanks

Lee Sigelman

Howard J. Silver

William O. Slayman

Paul M. Sniderman

Mary E. Stuckey

Charles V. Walker

Kenneth N. Waltz

James Q. Wilson

Thomas R. Wolanin

Yale University, The

MacMillan Center and

Department of Political

Science

Nancy H. Zingale

\section{BUILDERS CIRCLE}

$(\$ 2,500+)$

Anonymous

Alan I. Abramowitz

David Adamany

James E. Anderson

Gary J. Andres

Patricia Artinian

Manuel Avalos

William I. Bacchus

Michael A. Baer

Paul Allen Beck

John F. Bibby

Amy B. Bridges

Michael A. Brintnall

Charles S. Bullock, III

Margaret Cawley

Philip E. Converse

Thomas E. Cronin

William J. Daniels
Christopher J. Deering

Jorge I. Dominguez

Lawrence C. Dodd

Marion E. Doro

Melvin J. Dubnick

Eastern Michigan University

Leon D. Epstein

Kathleen A. Frankovic

John A. Garcia

George J. Graham, Jr.

Virginia H. Gray

Joel B. Grossman

Charles D. Hadley

Harold D. Lasswell Society

John P. Harrod

Mary Hepburn

Anne H. Hopkins

Robert Huckfeldt

Karen M. Hult

Patricia W. Ingraham

M. Kent Jennings

Robert Jervis

Loch K. Johnson

Bryan D. Jones

Charles O. Jones

Juan March Institute

William J. Keefe

Herbert Kitschelt

Allan Kornberg

Kay Lawson

Avery Leiserson

Margaret Levi and Robert Kaplan

Burdett A. Loomis

Susan A. MacManus

David B. Magleby

Sheilah Mann

Thomas E. Mann

Jane Mansbridge

David R. Mares

Joel Margolis

David R. Mayhew

Daniel A. Mazmanian

Eileen McDonagh

Lorraine M. McDonnell and M. Stephen Weatherford 
Nancy R. McWilliams

Melissa R. Michelson

Michigan Public Management Institute

Jeffrey R. Miller and Margot L. VanDis

Karen O'Connor

David Scott Palmer

Leonard Parkinson

Desiree S. Pedescleaux

Gerald M. Pomper

Mary Cornelia Porter

James J. Prestage

George H. Quester

Beryl A. Radin

Ben F. Reeves

Randall B. Ripley

David B. Robertson

Alan Rosenthal

Kay Lehman Schlozman

Mildred A. Schwartz

Gary M. Segura

Roberta S. Sigel

Barbara Sinclair

Rogers M. Smith

Raphael J. Sonenshein

John D. Sprague

Harvey Starr

Joseph Stewart, Jr.

Judith H. Stiehm

James A. Stimson

Camilla M. Stivers

Clarence N. Stone

Edward Thompson III

James A. Thurber

Sue Tolleson-Rinehart

Toni-Michelle C. Travis

David B. Truman

University of California, Los Angeles, International Institute

University of Oslo, Foundation for Applied Research

Sidney Verba

John C. Wahlke

Charles E. Walker

Paul J. Weber

Susan Welch

Lois R. Wise
Women's Caucus for Political Science

Maurice C. Woodard

SECOND CENTURY SOCIETY

$(\$ 1,000+)$

Martha A. Ackelsberg

Herbert E. Alexander

Hayward R. Alker

Gabriel A. Almond

Eugene J. Alpert

American Review of Public Administration

Anonymous

Herbert B. Asher

David Austen-Smith

Judith A. Baer

Maryann Barakso

Samuel H. Barnes

Harold F. Bass

Frank R. Baumgartner

Samuel H. Beer

Jeffrey M. Berry

Henry Bienen

James W. Bjorkman

Christopher J. Bosso

Janet M. Box-Steffensmeier

Richard W. Boyd and Martha Crenshaw

John Brademas

Steven J. Brams

Richard A. Brody

Nancy Brune

Frances Burke

James MacGregor Burns

California Community Foundation

Elizabeth Capell

Lief H. Carter

Gretchen G. Casper

Leonard Champney

Eugene Y. S. Chang

Daniel S. Cheever

Richard Cheney

The Claremont Institute

Clarke E. Cochran

Louise K. Comfort

M. Margaret Conway

Martha Crenshaw

Milton C. Cummings, Jr.
Robert A. Dahl

Vincent and Anne Davis

Rodolfo O. de la Garza

Richard E. DeLeon

Patrick J. Deneen

Louis DeSipio

Christine Di Stefano

Thomas R. Dye

George C. Edwards III

Maria J. Falco

Jane E. Fountain

Linda L. Fowler

H. George Frederickson

William E. Frenzel

Jerry Friedheim

Beth C. Fuchs

Jurg Martin Gabriel

F. Chris Garcia

Lisa Garcia Bedolla

Louis C. Gawthrop

Samuel K. Gove

Glen A. Halva-Neubauer

Susan Webb Hammond

Roderick P. Hart

Mary Hawkesworth

Haworth Press, Inc.

Jeffrey R. Henig

C. Randall Henning

Rodney E. Hero

Paul S. Herrnson

Kim Quaile Hill

John F. Hoadley

Ole R. Holsti

J. Woodford Howard, Jr.

Robert J. Huckshorn

William E. Hudson

Robert P. and Dixie S. Huefner

Dennis S. Ippolito

Bruce W. Jentleson

Calvin C. Jillson

Mark P. Jones

Nolan E. Jones

Michael A. Jones-Correa

Jane Y. Junn

Melvin A. Kahn

Stanley Kelley, Jr.

Rita Mae Kelly

Samuel Krislov and Judith Gillespie
Janet M. Laible

Thomas P. Lauth

David L. Leal

David C. Leege

Gregory B. Lewis

Seymour Martin Lipset

Gerhard Loewenberg

Linda Lopez

Nancy S. Love

L. Sandy Maisel

Melanie Frances Manion

Benjamin Marquez

Janet M. Martin

Karen M. McCurdy

Anna and Richard L. Merritt

William Mishler

Charles L. Mitchell

Robert Montjoy

Christopher Z. Mooney

Lois B. Moreland

James A. Morone

Richard G. Niemi

Helmut Norpoth

Julie L. Novkov

John S. Odell

Kenneth T. Palmer

Glenn R. Parker

Carole Pateman

William D. Pederson

Nelson W. Polsby

Henry J. Pratt

Barbara A. Presnall

Michael B. Preston

Kenneth Prewitt

David E. Price

Philip L. Pritchett

Adam Przeworski

Nicol C. Rae

Lyn Ragsdale

Thomas F. Remington

Leroy N. Rieselbach

Bert A. Rockman

Ronald L. Rogowski

Richard and Rosemary Rose

Loren D. Ross

Donald S. Rothchild

Larry J. Sabato

Scott D. Sagan

Mark Q. Sawyer 
Thomas S. Schrock

Joanna Vecchiarelli Scott

Sally Coleman Selden

Donna E. Shalala

W. Phillips Shively

Christine M. Sierra

Dick W. Simpson

Theda Skocpol

Elliot E. Slotnick

Duncan Snidal

Robert J. Spitzer

Thomas A. Spragens, Jr.

Harold W. Stanley

Sybil L. Stokes

Gerald H. Stollman

Deborah Stone

Floyd E. Stoner

Jonathan R. Strand

Michael W. Suleiman

James L. Sundquist

Sidney Tarrow

Raymond Tatalovich

Elizabeth Theiss-Morse

Joan C. Tronto

George Tsebelis

University of California, Los Angeles, Institute of Industrial Relations

University of Houston, Department of Political Science

University of New Orleans, Department of Political Science

University of Wisconsin, Milwaukee Political Science Department

Arturo Vega

David Vogel

Charles E. Walcott

Robert S. Wallerstein

Walnut Foundation

Richard D. Warden

Mr. and Mrs. Lionel Washington

Kenneth Weakley

Herbert F. Weisberg

Clyde Wilcox

David M. Wilson

Graham K. Wilson

Raymond E. Wolfinger
Women's Caucus for Political Science, Western Political Science Association

Robert C. Wood

Deil S. Wright

Dali L. Yang

Martynas A. Ycas

Charles E. Young

James Sterling Young

James V. Young

Robert L. Youngblood

John R. Zaller

Joseph F. Zimmerman

ANNIVERSARY SOCIETY

(\$500+)

Anonymous

Joel D. Aberbach

Janet Lee Abrahm

Yvette M. Alex-Assensoh

Lisa Anderson

John W. Anderson

Christopher J. Anderson

Robert Axelrod

Lee Ann Banaszak

Pamela Bataillon

Lewis Bateman

Lawrence Baum

Seth B. Benjamin

Frances Stokes Berry

Gayle Binion

Janet K. Boles

Henry J. Bowers

M. Kenneth Bowler

Ann O'M. Bowman

Karlyn Bowman

Mitchell Brown

Carol Buckland

Emmett H. Buell, Jr.

Bruce J. Bueno de Mesquita

Nancy Burns

Gerard Patrick Bushell

Cambridge University Press

Michael P. Canning

David A. Caputo

Jason P. Casellas

Bruce E. Caswell

Janet M. Clark

David Collier

Joseph Cooper
Gary W. Cox

Charles Jeremy Curtoys

George Tinsley Davies

Michael C. Dawson

Linda C. Degutis

Delia E. Donatelli

Alexander A. Doska

Georgia Duerst-Lahti

Delmer D. Dunn

Cynthia H. Enloe

Victoria A. Farrar-Myers

Michael K. Fauntroy

Morris Fiorina

Lynne E. Ford

Christopher H. Foreman, Jr.

Jeffry A. Frieden

Kent Gardner

Michael Garrety

John B. Gates

Irwin N. Gertzog

Harvey Glickman

Marissa Martino Golden

Miriam A. Golden

Judith Lynn Goldstein

Kenneth Goldstein

Robert T. Golembiewski

Leonard E. Goodall

Charles W. Gossett

Robert C. Grady

Fred I. Greenstein

Jose Angel Gutierrez

Melinda Gann Hall

Susan B. Hansen

Carol Hardy-Fanta

Kerry L. Haynie

William A. Hazleton

Lenneal J. Henderson

Virginia S. Herring

Marjorie R. Hershey

Donald V. Hester

John R. Hibbing

Kim Hildred

Milton E. Hill

John W. Holcombe

Xiaobo $\mathrm{Hu}$

Evelyne Huber

Patricia A. Hurley

Vincent L. Hutchings

John Ishiyama
Tomoaki Iwai

Lawrence R. Jacobs

Harold K. Jacobson

Kenneth Janda

James Jennings

W. Landis Jones

Peter H. Juviler

Miles Kahler

Nancy Kassop

Karen M. Kaufmann

Margaret E. Keck

Sean Q Kelly

Mark A. Kessler

Donald F. Kettl

Anthony King

Samuel A. Kirkpatrick

Gary Klass

Robin A. Kolodny

Cynthia N. Ladd

David A. Lake

Peter Lange

Frances E. Lee

Logan A. Lee

Jan E. Leighley

Ralph Lerner

William F. Levantrosser

Mark I. Lichbach

Serge E. Logan

Robert Lorish

David Lowery

Arthur Lupia

Naomi B. Lynn

MacArthur Foundation

Stephen Macedo

Linda Kay Mancillas

Lynn Mather

Susan McAndrew

Alison Rios Millett McCartney

Scott D. McClurg

Nancy E. McGlen

Roger A. McGuire

Susan McWilliams

Cheryl M. Miller

H. Brinton Milward

Gwendolyn Mink

J. Donald Moon

Roy Morey

Robert J. Mundt 
Walter F. Murphy

Richard W. Murray

Roger B. Myerson

Richard E. Neustadt

Pippa Norris

Joseph S. Nye, Jr.

Rosemary O'Leary

Robert A. Packenham

Dorothy A. Palmer

Anne M. Parsons

Louis Pauly

Mark A. Peterson

Mark P. Petracca

Susan J. Pharr

Anne R. Pierce

John J. Pitney, Jr.

Philip H. Pollock III

Jay W. Prestage

Diane-Michele Prindeville

Andree E. Reeves

Chester D. Rhoan

Wilbur C. Rich

Erin E. Richards

Donald L. Robinson

John A. Rohr

Raymond K. Rossiter

Lloyd I. Rudolph

Susanne Hoeber Rudolph

Barry S. Rundquist

David R. Salazar

Anna Sampaio

Eve N. Sandberg

Virginia Sapiro

Jean Reith Schroedel

Ronald G. Shaiko

Mary L. (Molly) Shanley

Robert Y. Shapiro

Beth A. Simmons

Andrea Y. Simpson

Adaljiza Sosa-Riddell

R. Vladimir Steffel

Alfred C. Stepan

Randall W. Strahan

Kaare Strom

James H. Svara

C. Neal Tate

Norman C. Thomas

J. Ann Tickner

Jeffrey K. Tulis
Ashutosh Varshney

Barbara A. Vobejda

Kenneth D. Wald

S. Laurel Weldon

Jennifer Widner

Melissa S. Williams

David Wilsford

Rick K. Wilson

Frederick M. Wirt

Women's Caucus for Political Science: South

Kenneth K. Wong

Elisabeth Jean Wood

Pamela A. Zeiser

Michael P. Zuckert

\section{FRIENDS}

(\$250+)

Gabriele Abels

Robert B. Alexander

Stephen Amberg

J. Theodore Anagnoson

Kristi Andersen

Kevin Avruch

Q. Whitfield Ayres

Lisa Baldez

Donald G. Balmer

Matt A. Barreto

Edwina Barvosa

Christina Elizabeth Bejarano

Cristina Beltran

Jonathan Benjamin-Alvarado

Jeanne C. Blamey

Elizabeth T. Boris

MaryAnne Borrelli

Katya D. Bowers

James M. Brasfield

Brigham Young University

Stanley C. Brubaker

Jeremy Buchman

Donald J. Calista

Thomas M. Callaghy

Randall L. Calvert

David T. Canon

Susan J. Carroll

Karl H. Cerny

Sue Ellen M. Charlton

Roland Chi

John A. Clark

Susan E. Clarke
Myles L. Clowers

Celia F. Cohen

David L. Coleman

Christopher Colmo

William F. Connelly, Jr.

Constance E. Cook

Timothy E. Cook

William Corlett

David Covin

Cary R. Covington

Marian L. Currinder

Russell J. Dalton

Michelle D. Deardorff

Linda deLeon

Martha Derthick

Brendan J. Doherty

Julie Dolan

Mary H. Durfee

Carter Edgeworth

Peter Eisinger

Lee Epstein

Amitai Etzioni

Timothy J. Feddersen

Malcolm M. Feeley

Frank Feigert

L. Boyd Finch

Jane Flax

Daniel B. Fleming

Michael P. Fleming

Mark N. Franklin

John G. Geer

Shirley M. Geiger

Joyce Gelb

Michael A. Genovese

Carl Gershman

Esther S. Goldstein

Jane A. Gordon

Bruce N. Gregory

Ashley C. Grosse

Clifford P. Hackett

Henry E. Hale

Peter A. Hall

Richard L. Hall

Welling Hall

Samuel Halperin

John W. Harbeson

Robert A. Heineman

Andrew P. Helene

Allen D. Hertzke
Peter T. Higgins

Erik P. Hoffmann

Raymond F. Hopkins

Janice H. Hopper

Robert F. Hopper

Juan Carlos Huerta

Serge Hurtig

Amaney Jamal

Ted G. Jelen

E. Terrence Jones

Marion R. Just

Peter J. Katzenstein

Kate M. Kaufer

William R. Keech

Scott Keeter

Catherine McArdle Kelleher

Frank Kessler

John Kincaid

Bruce C. Ladd, Jr.

Steve Lagerfeld

David D. Laitin

Robert E. Lane

Bruce A. Larson

Lawrence LeDuc

Wei-chin Lee

Howard H. Lentner

David M. Levitan

Jack S. Levy

Jacob T. Levy

Evan S. Lieberman

Pei-te Lien

Ann Chih Lin

Alice M. Litwinowicz

Cary E. Losson

DeWayne L. Lucas

Forrest Maltzman

Suzanne M. Marilley

Rachel M. McCleary

Stephen D. McDowell

Andrew S. McFarland

Wilson Carey McWilliams

Eugene Meehan

Melissa Buis Michaux

Toni P. Miles

Bruce Miroff

Devra Coren Moehler

Kristen Renwick Monroe

James P. Monroe

Vincent G. Moscardelli 
Elizabeth F. Moulds

Esther N. Mwangi

Katherine C. Naff

Hitoshi Namikawa

Eric J. Narcisse

Candice J. Nelson

Dale A. Neuman

New England Political Science Association

Lloyd G. Nigro

William Niskanen

Irfan Nooruddin

Susan S. Northcutt

Lord Norton of Louth

William L. Oakley

David M. O’Brien

Thomas R. Oliver

Bruce I. Oppenheimer

James L. O'Sullivan

Diana M. Owen

Benjamin I. Page

Thomas L. Pangle

Robert A. Pastor

Kelly D. Patterson

Bradley H. Patterson

Paul C. Peterson

Steven A. Peterson

Eric Plutzer

Frank Prindle

Doris Marie Provine

Donald P. Racheter

David Rayside

Jean C. Robinson

Mark Carl Rom

Barbara S. Romzek

Rodolfo Rosales

Laura Roselle

Frances McCall Rosenbluth

Jo Ellen Ross

John E. Rouse

Herbert J. Rubin

Andrew C. Rudalevige

Andrew Sabl

Sylvia G. Sanders

Christopher M. Sands

Arlene W. Saxonhouse

Kenneth F. Scheve

Gordon Schochet

Antoinette G. Sebastian
H. P. Secher

Mary C. Segers

Donald Share

Kenneth A. Shepsle

George C. Shipley

Peter N. Skerry, Jr.

Steven S. Smith

W. Rand Smith

Etel L. Solingen

William C. Spragens

Robert H. Stern

Laura Stoker

Scott Straus

Carl D. Sutton

Carol M. Swain

Duane H. Swank

Henrik Syse

Ross B. Talbot

John Thomas Tambornino

Kathryn Dunn Tenpas

Santa Algeo Traugott

University of Baltimore

Paul P. Van Riper

George Vernardakis

Jennifer Nicoll Victor

Kenneth Vogel

Mark C. Walker

Shirley Anne Warshaw

Gregory R. Weiher

Eric D. Weinstein

Ralph R. Widner

Leo A. Wiegman

Eddie N. Williams

Patrick J. Wolf

Thomas P. Wolf

Laura R. Woliver

Bang-Soon L. Yoon

M. Crawford Young

Eleanor E. Zeff

J. Nicholas Ziegler

\section{ASSOCIATES}

(\$100+)

Anonymous

Rahma Abdulkadir

David B. Abernethy

Henry J. Abraham

Paul M. Abrahm

Marisa Abrajano

Paul R. Abramson
Samer abu Libdeh

Brooke A. Ackerly

Carl D. Adrianopoli

Shariff Ahmad

Oluwaseyi Sherifat Akinleye

Roberto Alejandro

John R. Alford

Gar Alperovitz

Mohammed Alsaud

James E. Alt

Sharon Z. Alter

Jessica Racine Altif

Saladin Malik Ambar

Jennifer Amyx

Anthony A. Anderson

Stanley V. Anderson

Ingrid D. Anderson

Krister P. Andersson

Michael Lee Andrews

Melvin T. L. Ang

Gloria A. Anthony

Murillo de Aragão

Zehra F. Kabasakal Arat

Asher Arian

James Armistead

Paul E. Arnold

Howard Arnould

Kenneth Arrow

Jaime Baeza Freer

Isaac D. Balbus

Thomas J. Baldino

Joseph J. Balitewicz

Benjamin R. Barber

William T. Barndt

Leah Bassel

Sharon L. Basso

Brady P. Baybeck

Staci L. Beavers

Karen Beckwith

Arnold Beichman

Lauren Cohen Bell

David J. Bellshaw

Jane Bendix

Elizabeth A. Bennion

Frederic A. Bergerson

David R. Berman

Nancy Bermeo

Terri Bimes

R. Kenton Bird
Donald Bishop

Pamela Blackmon

Janice L. Bockmeyer

Catherine Boone

W. James Booth

Eileen Hunt Botting

Peter J. Bowman

Patrick T. Brandt

Sheri Breen

Christine Thurlow Brenner

Evelyn S. Brewster

Alan Brodman

Allan R. Brown

Benjamin Brown

Victor Bruce

Kent M. Brudney

Susan R. Burgess

John Francis Burke

Margaret Burns

Kenneth G. Busch

John W. Caldwell

Andrea Louise Campbell

Colin Campbell

David E. Campbell

James E. Campbell

Elliot W. Carlson

Ellen Carnaghan

Jason Caro

R. Charli Carpenter

Thomas M. Carsey

N. Joseph Cayer

Tyson Chaney

Jeffrey E. Cohen

Howard A. Cohen

Sally S. Cohen

Kenneth M. Coleman

Deirdre M. Condit

Margaret R. Connelly

Charles E. Cook, Jr.

Michael J. Coppedge

Ross J. Corbett

J. Kevin Corder

David Corvette

Joseph W. Cover

Michael C. Craw

Lyn Loyd Creswell

Joseph Cropsey

William J. Crotty

Jose E. Cruz 
Mark C. Curtis

Christopher P. Dallas-Feeney

Fred R. Dallmayr

Charles U. Daly

Alfonso J. Damico

Rafaela Dancygier

David J. Danelski

James L. Danielson

Charles Robert Davidson

David W. Davis

R. G. Davis

Peter deLeon

Joel H. Delofsky

Kathryn G. Denhardt

I. M. (Mac) Destler

Lisa J. Disch

Donna Robinson Divine

Richard E. Dodson

Kathleen Dolan

David P. Dolowitz

David B. Dorman

Jennifer J. Douglas-Abubakar

Michael W. Doyle

Murray P. Dry

Francis J. Duggan

Claudia Hastings Dulmage

Thomas L. Dumm

Pat M. Dunham

James N. Edelman

Catherine R. Edwards

Jennifer Einspahr

Richard C. Elling

Harold E. Engle

Kenneth Paul Erickson

David F. Ericson

C. Lawrence Evans

Alfred B. Evans, Jr.

Jana Everett

Angelo Falcon

Robert K. Faulkner

Margaret Kent Feczko

Paul Ferber

Raquel Fernandez Garcia

James W. Fesler

Elliott Fiedler

James E. Fleming

James S. Fleming

Frederic J. Fleron, Jr.

Heath Fogg Davis
Donald L. Fowler, Sr.

Jonathan A. Fox

Richard L. Fox

Lorraine W. Frank

Robert J. Franzese, Jr.

Siegrun Fox Freyss

Robert S. Friedman

Amy Wallerstein Friedman

Ester Rachel Fuchs

Archon Fung

Paul J. Furiga

Fritz Gaenslen

Henry C. Galant

William A. Galston

Judith A. Garber

Herbert Garfinkel

John P. Geis II

Thomas R. Genton

Michael Gerard

John Gibbons

Clark C. Gibson

Ernest D. Giglio

Micheal W. Giles

Emily R. Gill

Clare Ginger

Girls Ranch, Inc. of Arizona

Marianne Githens

Holly T. Goerdel

Brian David Goldberg

Sheldon Goldman

Eric Gonzalez Juenke

Jeff Goodwin

George J. Gordon

Nicole A. Gordon

Amy D. Gossett

Peter A. Gourevitch

Joanne Gowa

Claus Gramckow

Jim Granato

Wyn P. Grant

Hannah S. Greenberg

Joseph M. Grieco

Colleen M. Grogan

Christian R. Grose

Martin Gruberg

Anna M. Grzymala-Busse

Dagmar Gunther-Stirn

Michael K. Gusmano

Mary E. Guy
Todd Haltiner

Mary R. Hamilton

Lori Cox Han

Joseph F. Harkins

S. Suzan J. Harkness

Shiela Harmon-Martin

Lawrence Harrison

Patricia Harte

Roger E. Hartley

Virginia Haufler

Chip Hauss

Jennifer P. Haydel

Robert L. Healy

Ken Heath

John P. Heinz

Francis H. Heller

Charles P. Henry

Paul R. Hensel

Mark J. Herbst

Margaret G. Hermann

Richard Herrera

Alisa Hicklin Fryar

Ben Highton

Leslie I. Hill

H. N. Hirsch

Daniel J.B. Hofrenning

Kay S. Holcombe

Kenneth R. Hoover

Randolph C. Horn

Stephen Horn

R Gordon Hoxie

Margaret S. Hrezo

Constance C.T. Hunt

Leann Hurst

Gary Hymel

Matthew M. Incera

Helen M. Ingram

Yoshikazu Iwabuchi

Alice M. Jackson

Sherry B. Jeffe

Jim Moss \& Company

Jack Johannes

Roberta Ann Johnson

Tobe Johnson

Jocelyn M. Johnston

Richard G.C. Johnston

Mary Donovan Jones

Ronald Kahn

Max M. Kampelman
Christopher F. Karpowitz

Junko Kato

Ellis Katz

Dimokritos Menelaos Kavadias

Thomas A. Kazee

Richard C. Kearney

Francis J. Keenan

Lael R. Keiser

Christopher Kelaher

Christopher B. Kenny

Samuel Kernell

Rogan Kersh

Vincent B. Khapoya

Gary King

Mae C. King

George M. Kingman

James L. Kingsland

Maureen Kirchhoff

Jennet Kirkpatrick

Jason F. Kirksey

John J. Kirlin

Kathleen Knight

Matthew A. Kocher

Mona Lena Krook

Michael G. Krukones

Richard Labunski

Marc Landy

Joseph H. Lane, Jr.

Thomas S. Langston

Joseph LaPalombara

Miriam J. Laugesen

Jessica L. Lavariega Monforti

Kathryn C. Lavelle

Thelma Lavine

Lan Thuc Le

Susan S. Lederman

Jerome S. Legge, Jr.

Valerie Lehr

Janet E. Leigh

Lance T. LeLoup

Arthur J. Lerman

Michael H. Levin

Meira Levinson

Roy Licklider

Jethro K. Lieberman

Carl Lieberman

Sarah F. Liebschutz

Michael Lienesch 
Tracy L. R. Lightcap

Thomas W. Likens

Keena Lipsitz

Soledad Loaeza

Marvin R. Loewy

Dianne N. Long

Andrea M. Lopez

Nicholas P. Lovrich, Jr.

Lynne Rienner Publishers

Roderick MacFarquhar

Stuart A. MacNiven

Kathleen A. Mahoney-Norris

Michael J. Malbin

Harvey C. Mansfield

Paul Christopher Manuel

Andrei S. Markovits

Andrew D. Martin

Donald J. Matthewson

Russell Mayer

Rick Mayes

Steven Maynard-Moody

Mark G. Mazzie

Fletcher McClellan

John P. McCormick

H. Coleman McGinnis

Scott L. McLean

Leslie B. McLemore

Jillian Medeiros

Tali Mendelberg

Arthur E. Menna

Carol A. Mershon

Wanda F. Meyer-Price

Kevin J. Middlebrook

Sidney M. Milkis

Gabriel Miller

Harris N. Miller

Helen V. Milner

Patrice Mitchell

Carla E. Molette-Ogden

Celeste M. Montoya

Lisa J. Montoya

Richard M. Moose

David R. Morgan

Lorenzo Morris

K.C. Morrison

Wynne Walker Moskop

Charles C. Moskos, Jr.

Ken Moskowitz

Burton B. Moyer, Jr.
Donald P. Moynihan

Susanne D. Mueller

William D. Muller

Costanza Musu

Akiko Nakamura

John Nalbandian

Carol R. Nechemias

Aram Nerguizian

Catharine Newbury

Kathryn Newcomer

Charldean Newell

David Newman

Daniel L. Nielson

Lela G. Noble

Melissa Nobles

Joseph L. Nogee

Barbara Norrander

Noelle Helen Norton

Grady H. Nunn

Elizabeth A. Oldmixon

Mark Oleszek

Laura R. Olson

Susan M. Olson

Molly O'Neal

Frederick K. Orkin

Sima R. Osdoby

Chieko Kitagawa Otsuru

Harry P. Pachon

Elizabeth Paddock

Marian Lief Palley

Roy Parker, Jr.

Christopher S. Parker

Samuel C. Patterson

Thomas E. Patterson

Jeffrey S. Peake

Edward Lewis Pederson

Pamela D. Pelletreau

J. Salvador Peralta

Anthony D. Perry

Jack Pfister

Pfizer Inc.

John R. Phillips

John Charles Pierce

Neil Andrew Pinney

Richard M. Pious

M. Anne Pitcher

Jerry L. Polinard

Linda O. Polsby

Portal Pine Creek Canyon
Marsha Pripstein Posusney

Stephen Poulson

G. Bingham Powell, Jr.

Princeton University

Wendy M. Rahn

Hal G. Rainey

Ronald B. Rapoport

Sharon LaWanda Rapport

Jorgen S. Rasmussen

James H. Read

David P. Rebovich

Mavis Mann Reeves

Andrew Rehfeld

Rob Reich

Beth Reingold

Howard L. Reiter

Russell D. Renka

Stanley A. Renshon

Joao Resende-Santos

William G. Resh

Norma M. Riccucci

Ross R. Rice

Lilliard E. Richardson, Jr.

Julia T. Richie

Irelene P. Ricks

Elizabeth Rigby

Alice Robbin

James C. Roberts

Pearl T. Robinson

Scott E. Robinson

Margaret A. Robinson

Maya Rockeymoore

Robert R. Rodgers

John E. Roemer

Susan Carol Rogers

Meredith Rolfe

William J. Ronan

Roger P. Rose

James N. Rosenau

Marie B. Rosenberg

Nancy L. Rosenblum

Cindy Simon Rosenthal

Howard Rosenthal

Margaret Ann Rosenthal

Lee Ross

Marc Howard Ross

Francis E. Rourke

Blair A. Ruble

Ricardo Antonio Ruiz de la Serna
Sage Publications

Motohide Saji

Stephen G. Salkever

Kira Sanbonmatsu

Marjorie Sarbaugh-

Thompson

Eric Schickler

Steven E. Schier

Andrew J. Schlewitz

Patricia Schlueter

John R. Schmidhauser

Anne L. Schneider

Mark L. Schneider

Edward Schneier

Cheryl M. Schonhardt-Bailey

Morton Schoolman

Joseph M. Schwartz

Carl E. Schwarz

Robert Scigliano

Harold Seidman

Paul J. Seligman

Robert Serec

Jeffrey G. Seward

D. Michael Shafer

Byron E. Shafer

David M. Shafie

Henry A. Sheinkopf

Tommie Shelby

Mack C. Shelley II

Kenneth Sherrill

Doh C. Shin

Colleen J. Shogan

Carolyn M. Shrewsbury

W. Scott Shrewsbury

Steven A. Shull

Jeffrey Sikkenga

Elaine E. Silverman

Sheldon W. Simon

Lewis B. Sims

Henry B. Sirgo

Royal Daniel Sloan, Jr.

Corwin E. Smidt

Daniel A. Smith

Edmond C. Smith

Elizabeth S. Smith

Kevin B. Smith

Robin Smith

Stephen Samuel Smith

Bonnie Smith Whyte 
Leonid V. Smorgunov

Albert Somit

Roy A. Speckhard

Leonard M. Sperry, Jr.

Robert L. Spurrier, Jr.

Joseph L. Stager

Robert T. Starks, Emeritus

Manfred B. Steger

Michael Bernard Stein

Carl W. Stenberg III

Otis H. Stephens

Arthur R. Stevens

Andrea Stevenson Sanjian

Charles Stewart III

George Stickles

Susan C. Stokes

Walter J. Stone

Jeffrey D. Straussman

Mervin K. Strickler, Jr.

Philippa Strum

Henry Suhr

Terry Sullivan

Todd Swanstrom

Martin J. Sweet

Ann Swidler

Ivan Swift

Mina Tadrus

Lorraine M. Tafoya

Joseph M. Tages II

Donald G Tannenbaum

Raymond Tanter

Kathleen Tapport

Nathan Tarcov

Jennifer Morrison Taw

A. Stephen Telkins

Morton J. Tenzer

The Freedom Forum

Leslie Paul Thiele

Craig W. Thomas

Emma S. Thomas

Sue Thomas

Frank J. Thompson

Joan Hulse Thompson

Kurt M. Thurmaier

Betty Tillman

Leslie A. Tourigny

Peter Trubowitz

Gary Tschoepe

Dale Turner
Brian C. Turner

Jack Turner

Sean W. Twombly

Frank J. Tysen

Otto F. Unsinn

R. Lawrence Vandenberg

Susanne E. Vandenbosch

Kenneth L. Verdoia

Yaacov Y.I. Vertzberger

Thomas Vocino

Craig Volden

Helen R. Voss

David B. Walker

Thomas G. Walker

Sherri L. Wallace

Katherine Cramer Walsh

Hanes Walton, Jr.

Herbert Waltzer

Yanlai Wang

Adam L. Warber

Michael D. Ward

Robert E. Ward

Price I. Watkins

William L. Waugh, Jr.

David J. Webber

Carola Weil

David M. Welborn

Andreas Wesserle

Ellis M. West

Bruce Western

Marcia Lynn Whicker

Gordon P. Whitaker

Stephen L. White

Terri White

Sharon G. Whitney

Donald Wickler

Curtis C. Wilkie, Jr.

Steven I. Wilkinson

Kim M. Williams

Elizabeth R. Wingrove

Deborah A. Wolf

Matthew D. Wolf

Sheldon S. Wolin

Stephen B. Wood

Michael J. Woodruff

Richard Worthington

J. Mark Wrighton

Mark Wynn

Takakazu Yamagishi
John E. Yang

Dvora Yanow

Barry Zalcman

Anne B. Zill

James M. Zoetewey

Aristide R. Zolberg

Alan S. Zuckerman

\section{CONTRIBUTORS}

\section{(\$1-99)}

Sabella Ogbobode Abidde

Christopher H. Achen

George F. Ackerman

Marsha E. Ackermann

Thomas W. Adams

E. Obiri Addo

Robert Agranoff

Takeshi Akiba

John H. Aldrich

Akmal Aleemi

Karen J. Alter

Winifred L. Amaturo

Greg Anderson

Stefan Andreasson

Yuen Yuen Ang

Gloria C. Anglon

Connie Anthony

Jacquelyn A. Anthony

Ciro Antignani, Jr.

Andrew L. Aoki

Dominique Apollon

Harriet B. Applewhite

Janni L. Aragon

Rogério Bastos Arantes

Kathleen M. Araujo

Phillip J. Ardoin

Luisa Maria Arevalo

Hadley Arkes

Koki Theodore Asakura

Michael A. Ashburn

Athanasios Augoustatos

Sharon Denise Austin

Austin College

Olga A. Avdeyeva

George F. Avelino

Claudia N. Avellaneda

Regina Axelrod

Brent E. Ayer

Carl Baar

Kimberly Bakeberg
John R. Baker

Nancy V. Baker

Navin A. Bapat

Twiley W. Barker, Jr.

Derek Barker

David M. Barrett

Michael J. Barron

Robert Barsley

Jay Barth

Richard M. Battistoni

Robert F. Bauer

Joyce A. Baugh

Bruce Baum

Donald C. Baumer

Ronald J Beaton

Nancy L. Bednar

Franz Beitzinger

Robert Earl Bender

Seyla Benhabib

Liza Abram Benham

Jane Bennett

Kenneth R. Benoit

Stanley P. Berard

Luke Angelo Bergamini

Allison Berland Kaul

William T. Bernhard

Nancy Berson

Wallace H. Best

Thomas A. Birkland

Robyn M. Bishop

Bidisha Biswas

Naomi Black

Lavonna J. Blair Lewis

Randall W. Bland

John W. Blaney

Rebecca E. Blanton

Eric R. Boehme

Charles R. Boehmer

Alison E. Bolt

Thomas J. Bond

Frank Bonilla

Thomas E. Borcherding

Linda Bosniak

Philip Duncan Bousquet

Patricia R. Bowley

Dawn Brancati

Gildo Marcal Brandao

Pamela T. Brannon

Kathleen A. Bratton 
Amanda Jean Brazier

John Brehm

Reuben E. Brigety II

Kel Jacobs Britvec

Nailah Brock

Evelyn Z. Brodkin

Roger A. Brooks

Deborah Jordan Brooks

John E. Brown

John R. Brown

Ronald E. Brown

Rufus P. Browning

J. Lawrence Broz

Daniel Brumberg

Anthony R. Brunello

J. Vincent Buck

Eloise A. Buker

Fran Lisa Buntman

Thawilwadee Bureekul

Thomas F. Burke

Ross E. Burkhart

Walter Dean Burnham

Peter F. Burns

Carolee Bush

Elizabeth Bussiere

Charles E. Butterworth

Keith J. Bybee

Deanna Caldwell

Direlle R. Calica

Pamela Camerra-Rowe

Joseph Cammarano

Christopher M. Cardona

John M. Carey

Douglas Carlisle

Sara G. Carpenter

Nancy Carrillo

R. Wesley Carrington

Berenice A. Carroll

Brenda W. Carter

K. Lynn Cates

Matthew M. Caverly

James W. Ceaser

Stefanie Chambers

Steve Charnovitz

Rumi Chen

Francis Cheneval

Michelle L. Chin

Robert C. Chope, Jr.

Barbara Ann Chotiner
Tom Christensen

Jeffrey Church

Allan J. Cigler

Caleb M. Clark

Jennifer Hayes Clark

Rosalee Clawson

Joan B. Claybrook

Frank R. Cleminson

David Clinton

J. Patrick Coby

William B. Cody

Cathy J. Cohen

Frank S. Cohen

Joshua Cohen

Gloria Cohen-Dion

Daniel Cohn

Guy C. Colarulli

Leonard A. Cole

Sylvester (SC) Coleman

Kenneth Collier

Freddie C. Colston

Michael W. Combs

Anand Bertrand Commissiong

Carol B. Conaway

Timothy J. Conlan

Edward Steven Connell

Pamela Johnston Conover

Pamela Y. Cook

Catherine M. Cooney-Mesker

Gary W. Copeland

Javier Corrales

Jeronimo Cortina

Thomaz G. Costa

Charles L. Cotrell

Cornelius P. Cotter

John W. Cotton

George H. Cox

Thomas Craemer

Neta C. Crawford

Sue E.S. Crawford

Ingrid Creppell

Michael Crespin

Jesse C. Crosson

Jason A. Crotty

Don W. Crowley

Jose Miguel Cruz

Paul J. Culhane

Michael S. Cummings

Timothy W. Cunningham
Richard T. Cupitt

Paisley Currah

George J. Cvejanovich

Eugene Davidson

Donn G. Davis

Linda Davis

Renee Noelle Day

Olavo L. P. de Carvalho

Thomas S. De Luca, Jr.

Matthew DeBell

Melissa Deckman

Rebecca E. Deen

David Dessler

Dieter Dettke

Bryan J. Dettrey

Mark G. Detweiler

John Michael Diamond

Larry Diamond

Christopher W. Diaz

Alberto Diaz-Cayeros

Charles K. Dilley

Michelle L. Dion

Kristin Smith Diwan

Daniel J. Doherty

Jameson W. Doig

Jaime Dominguez

T. J. Donahue

Matthew Doppke

Sandra Lee Dorociak

Paul Dosh

Charles W. Dougherty

James W. Douglas

Douglas C. Dow

Victoria Ann Doyon

Alex N. Dragnich

Anne L. Draznin

John S. Dryzek

Jane Duckett

Sean P. Duffy

Denise E. Dutton

Bradley F. Dyke

Valerie Earle

Cheryl L. Eavey

Alan O. Ebenstein

Marianne Eichelberger

Robert M. Eisinger

Ginger Leigh Elliott-Teague

Cali Mortenson Ellis

R. Amy Elman
Deborah K. Elms

Charles R. Epp

Arlen I. Erdahl

John G. Eriksen

Rodolfo Espino III

Lloyd S. Etheredge

J. Peter Euben

Diana Evans

Pamela S. Falk

Rick D. Farmer

David M. Farrell

Maureen C. Feeley

Stephen M. Feldman

Kathy E. Ferguson

Margaret R. Ferguson

Kenneth E. Fernandez

Sergio Fernandez

Isabel Anunciacao Ferraz

Camisao

Edwin H. Fields

Julio Figueroa Nunez

John W. Finch

Terence T. Finn

Alexander Christoph Fischer

M. Steven Fish

Samuel H. Fisher III

Robert Fishman

J. Samuel Fitch

Roy B. Flemming

Judy Casburn Floyd

Jackson B. Floyd

Edmund Fong

Lisa Pullen Foust

Pauldine France

Jason Frank

Frank Franz

Lorrie Frasure-Yokley

Frederick W. Frey

Verena Fritz

Fred M. Frohock

Timothy Frye

Lee Ann Fujii

Kichizo Fujimoto

Francis Fukuyama

Timothy Fuller

Frances Galante

Susan Gallagher

David H. Galler

Archibald Galloway 
Jorge M. Gonzales Galoffin

Rulon R. Garfield

R. Sam Garrett

Jose E. Garriga-Pico

Bryan Garsten

Robin Gaul

Raymond Gavins

Judith Gay

Scott Gehlbach

Myron Genel

Tracey E. George

Heather Getha-Taylor

Michael T. Gibbons

Anthony Gierzynski

Michael Jaye Gilbreath

Murray Gilford

Terry Gilmour

Jonathan Githens-Mazer

Peggy Glick

Robert L. Goerder

Catherine Goetze

Larry Gold

Leslie Friedman Goldstein

Mark Golub

Brenda Nicia Gonzalez Gomez

Vanna Antonia Gonzales

Naomi W. Goodell

Jeanette Goodstein

Ann Gordon

Kristin Goss

Mark A. Graber

Lawrence S. Graham

Wayne G. Granquist

Blake P. Graves

Margaret Gray

Anne Green

Donald P. Green

Christina M. Greer

Lewis K. Griffith

Bernard N. Grofman

Seth A. Grossman

Gerard S. Gryski

Nelson P. Guild

Catherine Blanche Guisan

Girish J. Gulati

Charles David Gustafson

Eugenio Guzman

Claire Haeg
Donald H. Haider

Donald P. Haider-Markel

Michael W. Hail

Susan Haire

Drew Philip Halevy

Brien Hallett

Leon Halpert

Mark Halverson-Wente

Roger Hamburg

Raymond A. Hamilton II

Dan J. Hansen

Christine B. Harrington

Dennis Harris

Fredrick C. Harris

Melissa V. Harris-Perry

Jonathan Hartlyn

Nancy Hartsock

Vivien Hastings

Leah Haus

Melissa A. Haussman

Daniel P. Hawes

Jonathan P. Hawley

Barbara J. Hayler

Eugene G Hayunga

Alice L. Hearst

Karen Hein, M.D.

Caroline Heldman

Laura A. Henry

Michelle E Hentz

Charles F. Hermann

Frederick M. Herrmann

Dean R Hewitt

Ronald S. Hikel

David B. Hill

Larry B. Hill

Paul T. Hill

Samuel M. Hines, Jr.

Hiroshi Hirano

Samuel B. Hoff

Stanley Hoffmann

Thomas M. Holbrook

Richard Samuel Holliday

Thomas T. Holyoke

Claudio A Holzner

Nadia Rabesahala Horning

Germaine A. Hoston

Peter B. Hovde

Christopher Howard

Susan E. Howell
Dustin Howes

Betty G. Hubbell

Martine Huberty

Larry L. Hudson

Alexandra Huneeus

Valerie F. Hunt

Robert E. Hunter

Kate Carney Huston

Philip Ingrassia

Giovanni Ingrosso

Antoine M. Jackson

Ambrous Jacobs

Erwin A. Jaffe

Jane S. Jaquette

Holly Jarman

Khoshaba Jasim

Amy E. Jasperson

Christina F. Jeffrey

Edward T. Jennings

Benjamin M. Jensen

Jennifer M. Jensen

Andres E. Jimenez

Michelle E. Johns

Avra Johnson

Douglas B. Johnson

Lori A. Johnson

Mark Johnson

Paul M. Johnson

Ollie A. Johnson

Vernon D. Johnson

W. Lee Johnston

Patrick S. Johnston

Bradford S. Jones

Murel M. Jones, Jr.

Charlotte Weizer Joseph

Peter Josephson

Carlos E. Juarez

Dennis R. Judd

Diana M. Judd

Zinaida Kally

Rumen Kanchev

Jason S. Kassel

Lyn Kathlene

Koji Kato

Herbert Kaufman

Karen M. Kedrowski

Jonathan Keller

J. Edward Kellough

Christine A. Kelly
Steven Kelts

Edward J. Kelty

Frederick Kent

Lane Kenworthy

Andreas Kern

John E. Kern

Mark Kesselman

Kouslaa Kessler-Mata

Gilbert M. Khadiagala

Dwight C. Kiel

Kent J. Kille

Doo-Rae Kim

So Young Kim

Loren A. King

Vanessa King

Sue W. Kirkpatrick

Knut Kirste

Elizabeth E. Kiss

Casey A. Klofstad

Robert D. Knight

Hidetaka Kobayashi

Stephen A. Kocs

Edward A. Kolodziej

Tomas Koontz

Fae L. Korsmo

Laura Kosch-Cocozzella

Thad Kousser

Hanako Koyama

John F. Kozeletz

Vitaly A. Kozyrev

Henry Krisch

Ladis K.D. Kristof

Paul F. Kromer

Seldon M. Kruger

Sloane Rachael Kuney

Todd Kunioka

Susan Kunkle

Edward Kwon

Dean P. Lacy

Marianne Lado

Gordon Lafer

Andrew Lamas

Alan C. Lamborn

Thomas D. Lancaster

Pierre F. Landry

Robert W. Lane

Carol Langdon

Stephen Lange

Vickie Langohr 
William Tricot Laventhal

Geoffrey C. Layman

Laureen Lazarovici

Loan K. Le

Victor T. Le Vine

Fragano S.J. Ledgister

Caitlin Harrington Lee

Chinyelu Kambui Lee

Taeku Lee

Carol Skalnik Leff

Gail Leftwich

Jeffrey W. Legro

Fabrice Lehoucq

Jean D. Lemasurier

Jonathan Lemco

Leany Barreiro de Sousa Lemos

Harry A. Lenhart

Stephan Lesher

David Lessard

Elaine S. Levine

Traci M. Levy

Jeffrey B. Lewis

Jeremy R.T. Lewis

Steven B. Lichtman

Robert C. Lieberman

Phoebe S. Liebig

Earl W. Lindveit

Juan J. Linz

Charles H. Lipson

Rebecca Lisi

George T. Little

David William Livingstone

Flordeliz Llarena

Jill L. Locke

Dennis E. Logue, Jr.

S. Hansen Long

George I. Lovell

Daniel H. Lowenstein

Joseph E. Lowndes

Carol R. Lubin

Ian S. Lustick

Lyceum Books, Inc.

Julia Lynch

Kit G. Machado

Fiona S. Mackay

Paasha Mahdavi

Barry Mahoney

Elaine P. Maimon
Elena Maltseva

Peter Phillips Mandaville

Ardith L. Maney

B. Gregory Marfleet

Michael Margolis

Walter G. Markham

Elizabeth Markovits

Irving Leonard Markovitz

Melissa J. Marschall

Sherry L. Martin

Jose Roberto Martinez

Helena Martins

Claude Marx

Curtis M. Masiello

John G. Mason

Roger D. Masters

Sharon H. Mastracci

Marsha A. Matson

Jeremy Mayer

Jamie Mayerfeld

Andrea McAtee

Margaret M. McBeth

Michael W. McCann

Allan L. McCutcheon

Eric L. McDaniel

Eric McGhee

Alexis McGill

Mary A. McHugh

David McIlvain

Lynn McKee

Clyde D. Mckee, Jr.

Heather Elko McKibben

Joan E. McLean

Roberta Medford

Stephen E. Medvec

James P. Melcher

Mark Mellman

Richard H. Melton

Charles E. Menifield

Bryan K. Mercier

Stephen A. Merrill

Martha L. Merritt

Tamara Metz

William Mezzetti

Elizabeth B. Miller

Joshua I. Miller

Mark C. Miller

Sven Miller

Terry L. Miller
Daniel J. Minnich

Dinshaw Mistry

Karen L. Mitchell

James Andrew Mitchell

Olivier R. Mitterand

Kimberly Moloney

Benoit François Monange

Mahmood Monshipouri

Tonia R. Moore

Frank O. Mora

Andrea L. Morato-Lara

Sarah M. Morehouse

Alejandro Moreno

Michael E. Morrell

Karen Mossberger

Russell Muirhead

Patrick A. Mulloy

Naomi Murakawa

Dale D. Murphy

Robert E. Mutch

Tun Myint

Stuart S. Nagel

Tommaso Nannicini

Jennifer Nedelsky

Joan M. Nelson

Dalmas H. Nelson

Sheila M. Neroda

Gerald Neumark

Joan Hillebrand Neumiller

Bijoux Gidibu Ngwanda

Cynthia T. Nickel

Katherine Niemiec

Demetra Smith Nightingale

Peter Nitschke

Kiyoko K. Nitz

Jill Norgren

Stephen Noriega

Stephen A. Nuno

Ikenna Jesse Nwoke

Sulayman Nyang

Anthony Martin O'Halloran

Stephen Wayne Okel

Chika Okoye

Brendan O'Leary

Mitchell A. Orenstein

B. D'Andra Orey

Anna Maria Ortiz

James S. Pacy

Lilian M. Pagan
Anthony Palmer

Costas Panagopoulos

Joseph B. Parker

Daniel J. Parks

Janine A. Parry

Ifechukwu Ajuta Paul

Arthur C. Paulson

Darryl C. Payne

Kathryn Pearson

Francisco I. Pedraza

John P. Pelissero

Jose Raul Perales

Moises Perez, Jr.

Clarisa Perez-Armendariz

Ellis Perlman

Ayodeji Kamau Perrin

Enrique Peruzzotti

Rosalind P. Petchesky

V. Spike Peterson

M.J. Peterson

Edward J. Pfeiffer

Justin Phillips

Brian J. Phillips

Brian Kristopher Pinaire

Jennifer Pitts

Raymond Polin

Andrew J. Polsky

Daniel R. Popkey

Gregory Portillo

Edward B. Portis

Brian L. Porto

Robert K. Postic

Mahendra Prasad

Robert Press

Melanye Tarea Price

Colin Provost

Kevin F. Quigley

David L. Rabin

Eric W. Rader

Bahram Rajaee

Rosemary Ramsey

Harry H. Ransom

James P. Rausch

Earl C. Ravenal

Sam W.P. Rea, Jr.

Arthur J. Reiger

Paul Rejai

Estanislao Rengifo

Joachim Karl Rennstich 
Bradley M. Richardson

Peter F. Riehm

Neal Riemer

Mary C. Riggall

Shelley Rigger

Joan Dunne Rittenhouse

Suzanne M. Robbins

Tracie Breshun Roberson

Kenneth M. Roberts

Ryan Robertson

Donald W. Robinson

Javier M. Rodriguez

Juan Carlos RodriguezCordero

George K. Romoser

Harlan Rosacker

Maria Rosales

Guillermo Rosas

Peter Rosegg

Raymond A. Rosenfeld

Bernard H. Ross

Robert L. Ross

Brandon Rottinghaus

Jason P. Rouby

Marya Rozanova

Stephen L. Rozman

Raul L. Rubio

Marva L. Rudolph

Henry Ruempler

Isabel Ruiz de Castañeda

Wilma Rule

Benjamin Joseph Rusek

James Taylor Rushing

Alan Ryan

Cynthia K. Ryan

Rodrigo Saavedra

Jennifer Schenk Sacco

Lawrence Saez

Robert C. Sahr

Sebastian M. Saiegh

Debra J. Salazar

Idean Salehyan

Jose R. Sanchez

Joe H. Sanchez, Jr.

Arthur Sanders

Elizabeth Sanders

Ellis Sandoz

Charles R. Venator Santiago

Ashutosh Sarker
Rodolfo Sarsfield

Patricia Saulsbery

Jill E. Savage

Laura J. Scalia

Nicole A. Scarborough

Beth S. Schapiro

Denise L. Scheberle

Abigael Schirmann

Vivien A. Schmidt

Hans Peter Schmitz

Mark Schneider

Sanford F. Schram

John M. Schroder

Philip A. Schrodt

Mark D. Schwartz

Brooke Michele Sciotti

Judithanne Scourfield McLauchlan

Stephen Scroggs

Lyle A. Scruggs

Steven Seidman

Deniz Senol Sert

Dharma Bahadur Shakya

Raeann Shane

Susan Heilbrunn Shapiro

Michael J. Shapiro

Todd C. Shaw

L. Earl Shaw, Jr.

Sarah Shea

Susan J. Shearon

Shawn Shieh

Masaki Shigeta

Harold B. Shill

Charles R. Shipan

David A. Shirk

Eleonora Siliprandi

Evelyn M. Simien

J. P. Singh

William J. Skane

Susan Navarro Smelcer

Daniel E. Smith

Gary Duane Smith

Travis D. Smith

J.H. Snider

Richard Sobel

David C. Sobelsohn

Femi Razaq Sobowale

Dorothy J. Solinger

Rosemary Glass Spalding
June S. Speakman

Jeff Spinner-Halev

Katherine Spring

William D. Stanley

Marek D. Steedman

Wayne P. Steger

Peter J. Steinberger

Sven Steinmo

Mark C. Stephan

Alonzo T. Stephens

Linda S. Stevenson

Kendra B. Stewart

John G. Stewart

Donald E. Stokes

Connie S. Stoner

Tracy B. Strong

Ricardo Sucre Heredia

Mansoor Suhail

Vickie B. Sullivan

Eileen M. Sullivan-Marx

Mary Summers

Ekaterina Svyatets

Robert H. Swansbrough

Rex J. Swartz

David Swickard

Michael Tabuwe-Aletum

William A. Taggart

Susette M. Talarico

Caitlin Talmadge

Jeremy Tamsett

Yuka Tanabe

Yasumasa Tanaka

Holley E. Tankersley

Aron G. Tannenbaum

Christina H. Tarnopolsky

Mark Zachary Taylor

James R. Terrell, Jr

Brandon M. Terry

Andrew Thangasamy

Gwynn Thomas

Martha Thompson

Steven Thompson

John T. Tierney

Alvin B. Tillery, Jr.

Seth P. Tillman

Barbara Tobias

Joanna Bache Tobin

Lars Toender

Constantine Trachanas
Andrea V. Traynham

Marc A. Triebwasser

Stacey Triplett

Francisco Jose Troconis

Betty W. Trotter

Chia-hung Tsai

N. Clyde Tucker

Robin L. Turner

Charles B. Turpin

U.S. Department of Health \& Human Services

Syed N. Uddin

Isaac Unah

University of North Carolina, Chapel Hill

Richard M. Valelly

Henry Valen

Angelo Valente

Ali A. Valenzuela

Arturo A. Valenzuela

Paul A. Vander Myde

Hovhannes R. Vardanyan

Helena Varela

Ivani Vassoler-Froelich

Jacqueline Vaughn

Ali Velshi

Jill Mccalla Vickers

Angel L. Viera-Tirado

Jose D. Villalobos

James Raymond Vreeland

Kevin Charles Wack

Richard Waghorne

Joel M. Wagoner

Lo Wing Wah

Jeremy Waldron

Thomas C. Walker

Diane E. Wall

Marc A. Wallace

Teresa T Walsh

Frederick Carl Walton

Yiwei Wang

Ann Ward

Jean Shumway Warner

Retha W. Warnicke

Charlotte B. Watson

Nadja Waxenegger

Marvin G. Weinbaum

Matthew Weinstein

Erika S. Weinthal

Laura C. Weir 
Margaret Weir

Danny G. Wells

Gerald T. West

Edward M. Wheat

William Whipple

Gregory W. White

Jean B. White

John Kenneth White

Ismail K. White

Jacqueline A. White

MisChele White

Roger W. White

Erik M. Wibbels

Nelson Wikstrom

Craig Steven Wilder

Harold L. Wilensky
Susan L. Wiley

Kay Williams

Linda Faye Williams

Leonard Williams

Mitchell R. Williams

Stewart Hall Williams

Thad Williamson

Eliza J. Willis

Ann R. Willner

David C. Wilson

Rick Wilson

Kenneth Alan Wink

Pamela Winston

John F. Witte

Jill N. Wittrock

Eugene Victor Wolfenstein
Janet L. Womack

Janelle Wong

James L. Wood

Patricia J. Woods

Neal Woods

Kristin Lynn Wormley

Fiona M. Wright

Steven T. Wuhs

Tao Xie

Blake Hunter Yagman

Kenneth S. Yalowitz

Masahiro Yamada

Ryusaku Yamada

Kazuo Yamauchi

Dong-Hoon Yang

Vickie Ybarra
Abigail M. York

Jeffery Allen Yothers, Jr.

Iris Marion Young

Jason Scott Young

Candace C. Young

Michele Zebich-Knos

Eric Zeemering

Diana D. Zentay

Yi Zhao

Brandon C. Zicha

Joseph Zikmund II

Thomas Zittel

Natascha M.E. ZowisloGrünewald

Tamas D. Zsitvay

Susanne Zwingel 

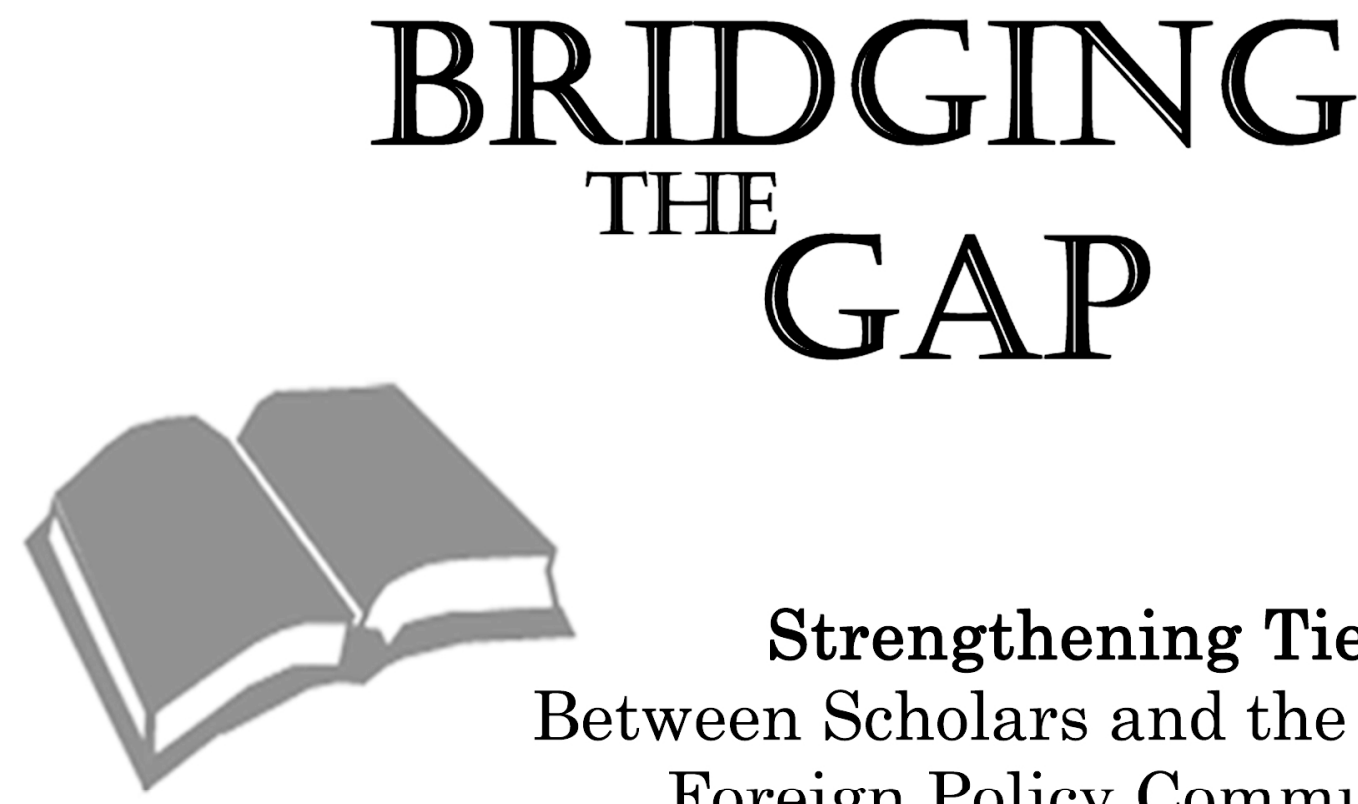

\section{Strengthening Ties Between Scholars and the Broader Foreign Policy Community}

Bridging the Gap seeks to connect faculty, graduate students, and policymakers in the international affairs and foreign policy communities to advance both research and careers.

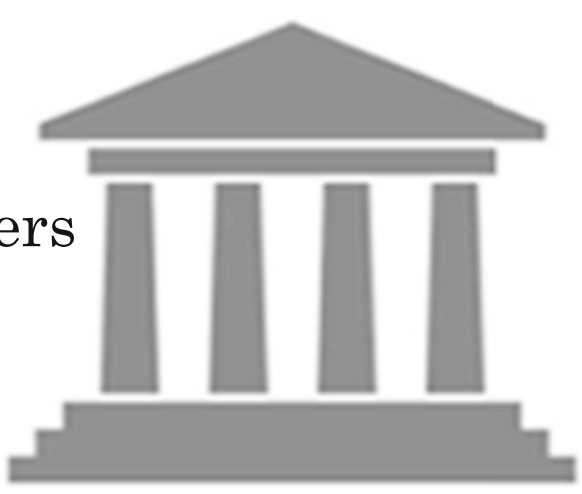

Learn about our three annual programs. Attend our reception at ISA (Thursday, April 4, 7:30 p.m., Continental 2)

Visit our webpage:

\section{Bridgingthegapproject.org}


Statement of Ownership, Management, and Circulation

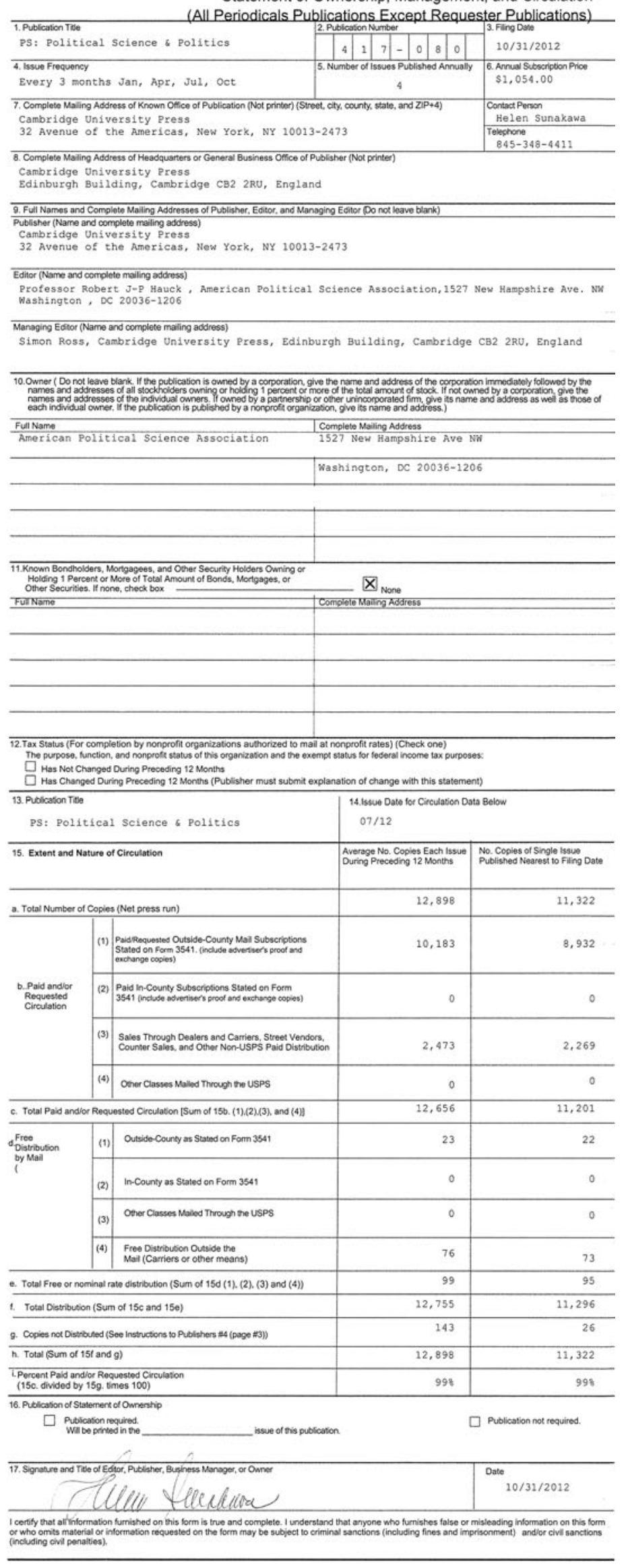

